We believe that our study is the first to identify different proportions of obstructive and restrictive airway disease between the main ethnic groups in sarcoidosis, and also the first to report an association of functional impairment with age. Despite a weak evidence base, sarcoidosis patients are often prescribed inhaled corticosteroids and $\beta$-agonists for respiratory symptoms regardless of their pattern of lung function at presentation [12]. Large airway obstruction in sarcoidosis is associated with increased mortality [13] and our data suggest that these patients may represent an under-diagnosed group. Further studies are needed to confirm these demographic associations, as well as to assess disease progression and response to inhaled therapies in this particular clinical phenotype of sarcoidosis.

\section{Thillai*, L. Potiphar*, C. Eberhardt*, M. Pareek*, R. Dhawan ${ }^{\#}$, O.M. Kon ${ }^{\#}$, M. Wickremasinghe", A. Wells ${ }^{\natural}$, D. Mitchell ${ }^{\top}$ and A. Lalvani*}

*National Heart and Lung Institute, Imperial College London, "St Mary's Hospital, and 'Interstitial Lung Unit, Imperial College London NHS Healthcare Trust, London, UK.

Correspondence: M. Thillai, Wright-Fleming Institute, National Heart and Lung Institute, Imperial College London, Praed Street, London W2 1NY, UK. E-mail: m.thillai@imperial.ac.uk

Statement of Interest: None declared.

Acknowledgements: We would like to thank S. Martin and the lung function team at St Mary's Hospital (Imperial College London NHS Healthcare Trust, London, UK) for their assistance with data collection.

\section{REFERENCES}

1 Handa T, Nagai S, Fushimi Y, et al. Clinical and radiographic indices associated with airflow limitation in patients with sarcoidosis. Chest 2006; 130: 1851-1856.
2 Baughman RP, Teirstein AS, Judson MA, et al. Clinical characteristics of patients in a case control study of sarcoidosis. Am J Respir Crit Care Med 2001; 164: 1885-1889.

3 Judson MA, Baughman RP, Thompson BW, et al. Two year prognosis of sarcoidosis: the ACCESS experience. Sarcoidosis Vasc Diffuse Lung Dis 2003; 20: 204-211.

4 Boros PW, Enright PL, Quanjer PH, et al. Impaired lung compliance and $\mathrm{DL}, \mathrm{CO}$ but no restrictive ventilatory defect in sarcoidosis. Eur Respir J 2010; 36: 1315-1322.

5 Tremblay A, Stather DR, Maceachern P, et al. A randomized controlled trial of standard $v s$ endobronchial ultrasonographyguided transbronchial needle aspiration in patients with suspected sarcoidosis. Chest 2009; 136: 340-346.

6 Jeebun V, Forrest IA. Sarcoidosis: an underrecognised cause for bullous lung disease? Eur Respir J 2009; 34: 999-1001.

7 Hankinson JL, Odencrantz JR, Fedan KB. Spirometric reference values from a sample of the general U.S. population. Am J Respir Crit Care Med 1999; 159: 179-187.

8 Sharma OP, Johnson R. Airway obstruction in sarcoidosis. A study of 123 nonsmoking black American patients with sarcoidosis. Chest 1988; 94: 343-346.

9 Agyemang C, Bhopal R, Bruijnzeels M. Negro, Black, Black African, African Caribbean, African American or what? Labelling African origin populations in the health arena in the 21st century. J Epidemiol Community Health 2005; 59: 1014-1018.

10 Baughman RP, Teirstein AS, Judson MA, et al. Clinical characteristics of patients in a case control study of sarcoidosis. Am J Respir Crit Care Med 2001; 164: 1885-1889.

11 Baughman RP, Nagia S, Balter M, et al. Defining the clinical outcome status (COS) in sarcoidosis: results of WASOG Task Force. Sarcoidosis Vasc Diffuse Lung Dis 2011; 28: 56-64.

12 Alberts C, van der Mark TW, Jansen HM. Inhaled budesonide in pulmonary sarcoidosis: a double-blind, placebo-controlled study. Dutch Study Group on Pulmonary Sarcoidosis. Eur Respir J 1995; 8: 682-688.

13 Viskum K, Vestbo J. Vital prognosis in intrathoracic sarcoidosis with special reference to pulmonary function and radiological stage. Eur Respir J 1993; 6: 349-353.

\title{
Reduced GM-CSF autoantibody in improved lung of autoimmune pulmonary alveolar proteinosis
}

\section{To the Editors:}

Pulmonary alveolar proteinosis (PAP) is a rare lung disease characterised by excessive accumulation of surfactant materials within alveolar spaces [1]. Patients with autoimmune PAP (aPAP) present a high level of granulocyte-macrophage colonystimulating factor (GM-CSF) autoantibodies (GM-Ab) in the serum as well as in bronchoalveolar lavage fluid (BALF) [2]. GM$\mathrm{Ab}$ neutralise the biological activity of GM-CSF in the lung [3], impairing terminal differentiation of alveolar macrophages and macrophage-mediated pulmonary surfactant clearance [4].

Based on the aetiology, clinical trials of exogenous GM-CSF supplementation have been carried out by a number of physicians with variable response rates ranging from 40 to $62 \%$ [5-9]. Previously, we reported that in three patients who received a pilot GM-CSF inhalation therapy, oxygenation was improved and the concentration of GM-Ab in BALF was reduced [7]. BONFIELD et al. [8] also reported that the serum titre of GM-Ab was reduced during successful treatment of aPAP with subcutaneously injected GM-CSF. However, our recent phase II trial of GM-CSF inhalation involving 35 patients revealed that serum levels of $\mathrm{GM}-\mathrm{Ab}$ remained unchanged throughout the therapy, suggesting that GM-CSF inhalation therapy did not affect the production of GM-Ab [9]. Thus, the effect of exogenous GM-CSF administration on GM-Ab levels in the serum remains controversial. This discrepancy may be due to differences in the route 
a)
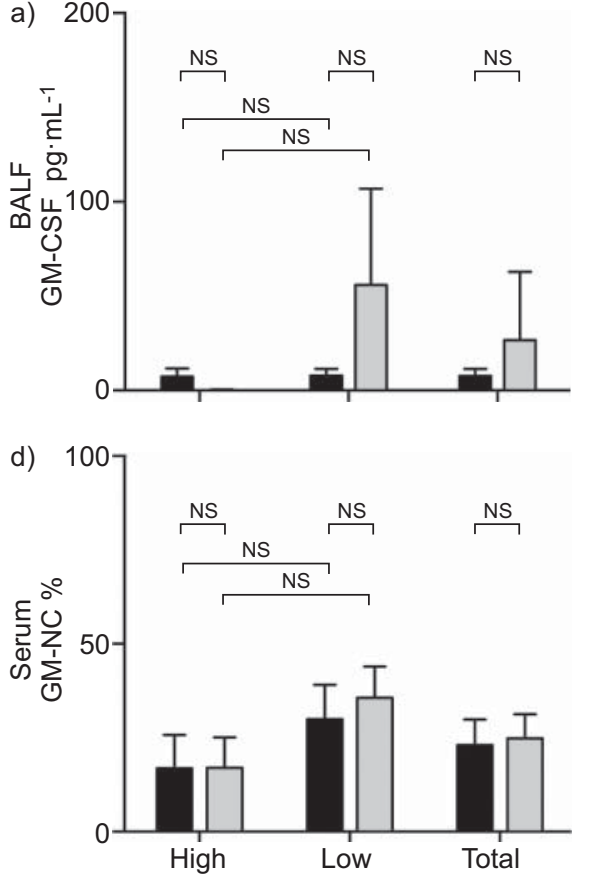

b)

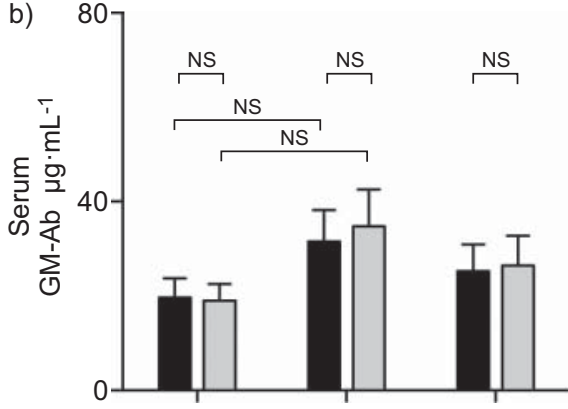

e)

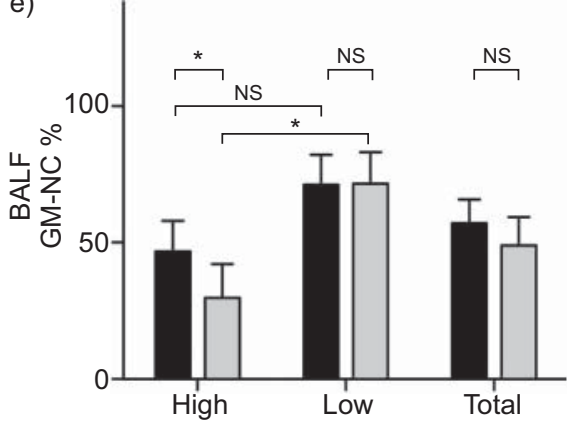

c)

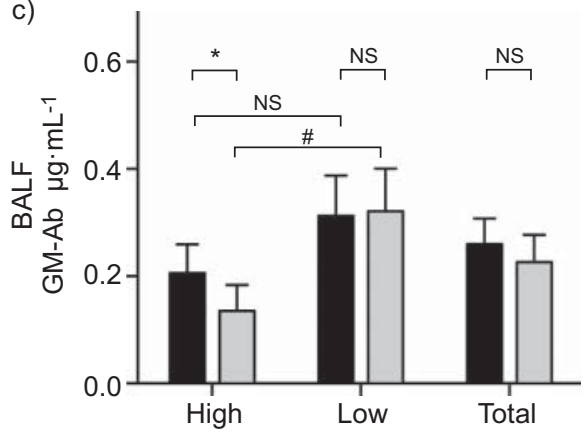

Before therapy

After therapy

FIGURE 1. The titres of granulocyte-macrophage colony-stimulating factor (GM-CSF) and GM-CSF autoantibodies (GM-Ab) in bronchoalveolar lavage fluid (BALF) and serum obtained from high and low responders and total patients before and after GM-CSF inhalation therapy. a) GM-CSF in BALF, GM-Ab b) in serum and c) in BALF, and GM-CSF-neutralising capacity (GM-NC) d) in serum and e) in BALF are shown. Data are presented as mean and SE. *: $p<0.05$; * $p=0.054$; NS: nonsignificant; $p$-values calculated using paired t-test for comparison between normally distributed data before and after therapy, and unpaired t-test for group comparison between high and low responders.

of administration and/or the dose of GM-CSF. Aerosolised GMCSF reaches the lower respiratory tract and may stimulate immature alveolar macrophages directly to promote terminal differentiation and improve the local clearance of the accumulated surfactant and GM-Ab, although it does not affect the production of systemic GM-Ab.

To test this hypothesis, we performed a retrospective study using preserved BALF obtained through an optional evaluation procedure from the patients that participated in a pilot study $(n=1)$ [7], an early phase II trial $(n=6)$ [9] and a multicentre phase

\begin{tabular}{|c|c|c|c|c|c|}
\hline TABLE 1 & \multicolumn{5}{|c|}{$\begin{array}{l}\text { Correlation between levels of granulocyte- } \\
\text { macrophage colony-stimulating factor (GM-CSF) } \\
\text { autoantibodies (GM-Ab) and GM-CSF- } \\
\text { neutralising capacity (GM-NC) in serum and } \\
\text { bronchoalveolar lavage fluid (BALF) }\end{array}$} \\
\hline \multirow[t]{2}{*}{ Variable } & & \multicolumn{2}{|c|}{$\begin{array}{l}\text { Before } \\
\text { treatment }\end{array}$} & \multicolumn{2}{|c|}{ After treatment } \\
\hline & & $\mathbf{r}^{\#}$ & p-value & $r^{\#}$ & p-value \\
\hline \multicolumn{2}{|c|}{ BALF GM-NC and BALF GM-Ab } & 0.7556 & 0.0005 & 0.8430 & $<0.0001$ \\
\hline \multicolumn{2}{|c|}{ BALF GM-Ab and serum GM-Ab } & 0.8616 & $<0.0001$ & 0.7780 & 0.0002 \\
\hline \multicolumn{2}{|c|}{ BALF GM-NC and serum GM-NC } & 0.5345 & 0.0271 & 0.6087 & 0.0073 \\
\hline
\end{tabular}

II trial $(n=12)$ [9] of GM-CSF inhalation therapy. Importantly, each patient underwent the bronchoalveolar lavage procedure on the same bronchus of the right middle lobe by the same operator of the same institute within 1 week of the start of, and after the end of, the GM-CSF inhalation therapy period according to the unified standard procedure protocol described previously [9]. BALF was sent to the Niigata University Medical and Dental Hospital (Niigata, Japan) and subjected to centralised analysis. As the median alveolar-arterial oxygen tension difference $\left(P A-a, \mathrm{O}_{2}\right)$ improvement was $13 \mathrm{mmHg}$, we classified the participants into two groups, high responders with an improvement $>13 \mathrm{mmHg}$ $(\mathrm{n}=10)$ and low responders with an improvement $<13 \mathrm{mmHg}$ $(n=9)$, in order to evaluate the relationship between therapeutic response and changes in the level of GM-CSF or GM-Ab in the serum and BALF. There were no significant differences between the two groups in symptoms, including cough and dyspnoea, demographic data, lung function tests, except for $\mathrm{PA}-\mathrm{a}, \mathrm{O}_{2}$, or BALF recovery rates.

To determine the stoichiometry of GM-CSF during the inhalation treatment, we measured the concentrations of total GM-CSF (i.e. autoantibody-bound plus free GM-CSF) in BALF according to a method described previously [10] in order to rule out the possibility that GM-CSF inhalation may augment the production of intrinsic GM-CSF in the lung. The concentration of total GMCSF did not change during the inhalation therapy in both high and low responders (fig. 1a). Thus, GM-CSF inhalation was not associated with an increase of GM-CSF in the lung. Notably, BALF of low responders tended to contain a high level of GMCSF that might derive from GM-Ab-GM-CSF complex residing in the alveolar space, as $>99 \%$ of serum GM-CSF was bound to 
GM-Ab [10]. The GM-Ab-GM-CSF complex might be incorporated into alveolar macrophages through $\mathrm{Fc}_{\mathrm{c}}$ receptors, which were remarkably reduced in aPAP [11], and thus the clearance of the complex was considered to be heavily impaired.

Consistent with our phase II study [9], the serum GM-Ab levels measured by ELISA [7] were unchanged during the treatment in both high and low responders (fig. 1b). The concentration in BALF, however, decreased significantly in high responders, but not in low responders after GM-CSF treatment (fig. 1c). The concentration tended to be higher in low responders than in high responders, but this was not statistically significant. Importantly, the mean molar ratios of GM-Ab to GM-CSF in BALF before and after GM-CSF inhalation were $2.6 \times 10^{4}$ and $4.9 \times 10^{4}$, respectively, indicating that most GM-Ab was capable of binding GMCSF in the lung.

The serum neutralising capacity against GM-CSF estimated using a GM-CSF-dependent cell line, TF-1 [10], was unchanged during the treatment in both groups (fig. 1d). However, the capacity was reduced in BALF obtained from high responders but not in low responders (fig. 1e). The decrease in BALF neutralising capacity during the treatment was probably due to the decrease in BALF GM-Ab concentration, because these two parameters significantly correlated with each other before and after the treatment (table 1). However, GM-Ab in the lung was considered dependent on circulating GM-Ab, because the concentration of GM-Ab and the neutralising capacity in BALF were closely correlated with those parameters in the serum before and after the treatment (table 1). Moreover, ratios of postto pre-treatment GM-Ab levels in BALF were strongly correlated with those of total immunoglobulin $G$ in BALF $(r=0.708$, $\mathrm{p}=0.0021)$, which significantly decreased $(\mathrm{p}<0.02)$ during GMCSF inhalation treatment. Taken together with the stable serum GM-Ab level during the treatment, the decrease in GM-Ab levels in the BALF of high responders is probably due to restoration of the local clearance capacity by terminally differentiated macrophages in the lung.

Since GM-CSF inhalation differs from subcutaneous administration in dose and administration route, mechanisms for therapeutic efficacy may differ between the two therapies. As indicated in this study, the amount of GM-CSF was far less than the amount of GM-Ab in the BALF and, therefore, it is unlikely that the inhaled GM-CSF bound to GM-Ab had directly contributed to the reduced concentration of GM-Ab detected by ELISA. Because pulmonary lesions of aPAP are typically distributed in a patchy manner, as indicated by the geographical pattern of ground-glass opacity in high-resolution computed tomography, inhaled GM-CSF may first reach the mildly affected pulmonary regions in the lungs and improve the dysfunction of alveolar macrophages at these sites. The functionally improved alveolar macrophages may contribute to promoting the clearance of accumulated surfactant and reducing the diffusion barrier, shunt fraction and/or ventilation-perfusion mismatching. Conversely, GM-CSF administered subcutaneously may bind to GM-Ab, and only a small part may directly reach the lungs. Most may reach the lymph nodes or bone marrow as immune complexes with GM-Ab that might be associated with immunological modulation, including suppression of autoantibody production.
In conclusion, we confirmed that GM-CSF inhalation was associated with a decrease of GM-Ab in the BALF in improved lungs, which was probably due to the restoration of clearance, and that GM-CSF inhalation might not affect autoantibody production. We believe that the data presented in this study enhance our understanding of the mechanism for effective GM-CSF inhalation therapy and may provide us with important information for determining the regimens of the treatment.

\section{K. Ohashi*\#, A. Sato ${ }^{\Uparrow}$, T. Takada\#, T. Arai $^{+}$, Y. Kasahara ${ }^{\S}$, M. Hojo ${ }^{f}$, T. Nei*, H. Nakayama*, N. Motoi*, S. Urano*, R. Eda**\#\#, M. Yokoba ${ }^{\text {ฯ }}$, Y. Tsuchihashi ${ }^{++}$, Y. Nasuhara ${ }^{\S \S}$,

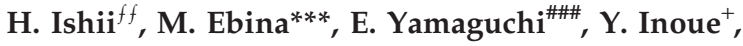 K. Nakata* and R. Tazawa*}

*Bioscience Medical Research Center, Niigata University Medical and Dental Hospital, "Division of Respiratory Medicine, Niigata University Graduate School of Medical and Dental Sciences, Niigata, ${ }^{+}$National Hospital Organization (NHO) Kinki-Chuo Chest Medical Center, Osaka, ${ }^{\S}$ Dept of Respirology, Graduate School of Medicine, Chiba University, Chiba, ${ }^{f}$ Division of Respiratory Medicine, National Center for Global Health and Medicine, ${ }^{f f}$ Dept of Respiratory Medicine, Kyorin University School of Medicine, Tokyo, ${ }^{* *} \mathrm{NHO}$ Yamaguchi-Ube Medical Center, Ube, ${ }^{\# \#}$ Kurashiki Municipal Hospital, Kurashiki, "Dept of Respiratory Medicine, Kitasato University School of Medicine, Kanagawa, ${ }^{++}$Institute of Tropical Medicine, Nagasaki University, Nagasaki, ${ }^{\S \S}$ First Dept of Medicine, Hokkaido University School of Medicine, Sapporo, ***Dept of Respiratory Medicine, Tohoku University Medical School, Sendai, \#\#\#Division of Respiratory Medicine and Allergology, Dept of Medicine, Aichi Medical University School of Medicine, Aichi, Japan, and "Dept of Pulmonary Biology, Cincinnati Children's Hospital Medical Center, Cincinnati, $\mathrm{OH}$, USA.

Correspondence: K. Nakata, Bioscience Medical Research Center, Niigata University Medical and Dental Hospital, 1-754 Asahimachi-dori, Niigata 951-8520, Japan. E-mail: radical@med.niigata-u.ac.jp

Support Statement: This work was supported in part by grants from the Japanese Ministry of Education and Science, Ministry of Health, Labour, and Welfare of Japan (H14-trans-014 to K. Nakata, H21-Nanchi-Ippan-161 to YI), Grant-in-Aid for Scientific Research (Category B 18406031 to Y. Inoue, Category C 22590852 to R. Tazawa), and National Hospital Organization of Japan (Category Network to Y. Inoue).

\section{Statement of Interest: None declared.}

Acknowledgements: The authors thank the investigators and patients who participated in this study, C. Kaneko and $\mathrm{H}$. Kanazawa for help with data management, M. Nakao and Y. Nakagawa for analyses of alveolar macrophages, and M. Mori for her help in preparing data for the manuscript (all from Bioscience Medical Research Center, Niigata University Medical and Dental Hospital, Niigata, Japan). 


\section{REFERENCES}

1 Rosen SH, Castleman B, Liebow AA. Pulmonary alveolar proteinosis. N Engl J Med 1958; 258: 1123-1142.

2 Kitamura T, Tanaka N, Watanabe J, et al. Idiopathic pulmonary alveolar proteinosis as an autoimmune disease with neutralizing antibody against granulocyte/macrophage colony-stimulating factor. J Exp Med 1999; 190: 875-880.

3 Seymour JF, Presneill JJ. Pulmonary alveolar proteinosis: progress in the first 44 years. Am J Respir Crit Care Med 2002; 166: 215-235.

4 Sakagami T, Beck D, Uchida K, et al. Patient-derived granulocyte/ macrophage colony-stimulating factor autoantibodies reproduce pulmonary alveolar proteinosis in nonhuman primates. Am J Respir Crit Care Med 2010; 182: 49-61.

5 Seymour JF, Presneill JJ, Schoch OD, et al. Therapeutic efficacy of granulocyte-macrophage colony-stimulating factor in patients with idiopathic acquired alveolar proteinosis. Am J Respir Crit Care Med 2001; 163: 524-531.

6 Venkateshiah SB, Yan TD, Bonfield TL, et al. An open-label trial of granulocyte macrophage colony stimulating factor therapy for moderate symptomatic pulmonary alveolar proteinosis. Chest 2006; 130: 227-237.
7 Tazawa R, Hamano E, Arai T, et al. Granulocyte-macrophage colony-stimulating factor and lung immunity in pulmonary alveolar proteinosis. Am J Respir Crit Care Med 2005; 171: 11421149.

8 Bonfield TL, Kavuru MS, Thomassen MJ. Anti-GM-CSF titer predicts response to GM-CSF therapy in pulmonary alveolar proteinosis. Clin Immunol 2002; 105: 342-350.

9 Tazawa R, Trapnell BC, Inoue $\mathrm{Y}$, et al. Inhaled granulocyte/ macrophage-colony stimulating factor as therapy for pulmonary alveolar proteinosis. Am J Respir Crit Care Med 2010; 181: 13451354.

10 Uchida K, Nakata K, Suzuki T, et al. Granulocyte/macrophagecolony-stimulating factor autoantibodies and myeloid cell immune functions in healthy subjects. Blood 2009; 113: 2547-2556.

11 Bonfield TL, Raychaudhuri B, Malur A, et al. PU.1 regulation of human alveolar macrophage differentiation requires granulocytemacrophage colony-stimulating factor. Am J Physiol Lung Cell Mol Physiol 2003; 285: L1132-L1136.

\section{Deficit of osteoprotegerin release by osteoblasts from a patient with cystic fibrosis}

\section{To the Editors:}

Cystic fibrosis (CF) is an autosomal recessive disorder caused by mutations of the CF transmembrane conductance regulator (CFTR), a cyclic adenosine monophosphate (cAMP)-dependent anion channel expressed mostly in epithelia. Bone deficiency is commonly seen in patients with $\mathrm{CF}$ and begins at a young age. Low bone mass affects children and young adults with CF and is associated with significant morbidity due to fractures and decreased lung function. Brittle bones in CF disease have been confirmed by densitometric data, the presence of fractures, and impaired quality of life of young and adult patients [1]. Whether or not this is caused by bone disease around puberty due to a poor acquisition of peak bone mass and worsens with age, lower bone mineral density (BMD) gains are already being observed in $\mathrm{CF}$ children with mild disease and normal nutritional status, suggesting that CF-related low BMD may, in part, be due to a primary defect in bone metabolism [2]. In human bone cells, the expression of CFTR protein has been identified by immunohistological observations [3]; we further reported the expression of CFTR mRNA and protein in primary human osteoblasts (the cells of bone formation) [4]. Although there is a report demonstrating a direct association between the F508del mutation and CF-related low BMD in young CF adults with at least one F508del allele [5], the effect of mutations in CFTR, specifically the F508del allele in bone cell metabolism is, to date, unknown.

Since its initial discovery in 1997 as a key regulator in bone density [6], osteoprotegerin (OPG), a product of osteoblasts, is now well known as an inhibitor of osteoclastogenesis. The OPG protein has been shown not only to inhibit osteoclast-mediated bone resorption, but also to exert direct osteoanabolic effects by increasing alkaline phosphatase activity and mineralisation in human osteoblasts [7]. To our knowledge, it is not known whether the F508del mutation in CFTR has a direct effect on human osteoblast activity.

Here, we report, for the first time, both defective CFTR-mediated chloride channel activity and a severe deficit of OPG protein release by osteoblasts of a 25 -yr-old CF male with the F508del/ G542X mutation in CFTR. The CFTR-mediated chloride channel activity and the level of OPG release were investigated in primary osteoblasts cultured from fresh ankle bone fragments from the CF patient. Normal human osteoblasts, used as controls, were obtained from fresh bone fragments of healthy young adults who underwent trauma surgery. All CF and normal primary osteoblast cell culture (used at the second to third passage and after confluence within 6-8 weeks) was performed as previously described [4]. The bone samples were obtained with informed patient consent after approval by the local research ethics committee (Faculty of Medicine, Reims, France).

First, to test the chloride channel activities in primary human osteoblasts, functional analysis was performed by measuring the cAMP-regulated and CFTR-dependent iodide efflux in the presence of a mixture composed of forskolin (an adenylate cyclase activator raising the cellular cAMP level) and genistein (a direct activator of CFTR), as previously described [8]. As shown in figure $1 \mathrm{a}$, a total absence of CFTR-dependent chloride response in F508del-CFTR osteoblasts was observed compared with normal osteoblasts. Interestingly, the two calcium-dependent and volumedependent chloride channels were found to be fully functional in both F508del-CFTR and normal osteoblasts. Secondly, we demonstrated that the level of both basal and stimulated (tumour necrosis factor- $\alpha, 20 \mathrm{ng} \cdot \mathrm{mL}^{-1}$ for $4 \mathrm{~h}$ ) release of OPG protein (evaluated by ELISA) in F508del-CFTR osteoblast cultures was approximately $8-10 \%$ of that observed in normal osteoblasts (fig. 1b). 